\title{
A Kind of Energy-efficient Routing Algorithm for WSN Based on HQEA
}

\author{
Lingxia $\operatorname{Liu}^{1} *$ and Qiang Song ${ }^{2,3}$ \\ ${ }^{1}$ Anyang Normal University, An'yang, 455000, China \\ ${ }^{2}$ Wuhan University of Technology, Wu'han 430073, China \\ ${ }^{3}$ Anyang Institute of Technology, An'yang, 455000, China \\ aysq168@163.com
}

\begin{abstract}
This paper proposes a new hierarchical routing algorithm for wireless sensor networks called hybrid QEA-based energy-efficient routing algorithm (HERA), which is based on LEACH and PEGASIS algorithms in the environment of wireless sensor networks. In order to reduce the data transmission distance, this algorithm uses the hybrid quantum evolutionary algorithm (HQEA) to establish the best cluster-based multi-chain topology. For the sake of balancing energy dissipation, node's residual energy and its distance from the target are considered as criterions of cluster head election, and each cluster head relays the sensed data of other clusters to the sink. Simulation results demonstrate comparing with LEACH and PEGASIS, this proposed algorithm HERA can shorten total transmission distance significantly, which is also more efficient to save and balance energy of consumption, meanwhile it prolongs the living time of whole network and eliminates the affection of sink's location on the network lifetime.
\end{abstract}

Keywords: Wireless sensor network, LEACH, PEGASIS, HQEA

\section{Introduction}

In the study of wireless sensor network routing algorithms, a hierarchical routing algorithm has more advantages than a planar routing algorithm [1], which can make the wireless sensor node to reduce its energy consumption and prolong survival time, thus make the whole network can work for a long time.

Currently, there are some researchers apply the intelligent optimizational algorithm to WSN routing protocol, to help WSN to look for the optimal network routing plan [4-7].

LEACH [2] and PEGASIS [3] are considered as the classic methods in a hierarchical routing algorithm to solve the problem of energy saving. Combining with the LEACH algorithm clustering topology and PEGASIS multiple jump chain communication mode, this paper puts forward a hybrid quantum evolutionary algorithm based on the high efficiency and energy-saving wireless sensor network routing algorithms, which keeps the advantages of LEACH and PEGASIS algorithms, abandons their deficiency; and through optimizing cluster head selection strategy makes topological structure more reasonable and the cluster head distribution more uniform, ultimately reduces energy consumption and makes the whole network survival period effectively improve. 


\section{System Description}

\section{1. Network Model}

This paper assumes that wireless sensor nodes are randomly distributed in a square target area. Sink is deployed out of the area, the energy is supposed infinite, the position of sink and sensor nodes are known and fixed, and nodes know their current surplus energy, wireless transmitting power can be adjusted according to the length of receiver's distance. According to the characteristics of network model in this paper, the method of combination of centralization and distribution is used to construct network routing link.

\subsection{Energy Model}

Wireless communication energy consumption is the main part in WSN, this paper uses the network energy consumption model in literature [2]. Sending $\mathrm{k}$ bits data to the position of distance $^{d}$, the energy consumption is:

$$
E_{T x}(k, d)= \begin{cases}k E_{\text {elec }}+k \varepsilon_{f s} d^{2} & d \leq d_{0} \\ k E_{\text {elec }}+k \varepsilon_{m p} d^{4} & d>d_{0}\end{cases}
$$

Inside the expression, $E_{\text {elec }}$ is the energy consumed by the wireless receiving and sending circuit. $^{\varepsilon_{f s}}$ is the energy ratio coefficient consumed by the signal amplifier when $d<d_{0}$, this time free space model is adopted, the energy loss is proportional to $d^{2} ;{ }^{\varepsilon_{m p}}$ is the energy ratio coefficient consumed by signal amplifier in the conditional of $d<d_{0}$, this time the multipath fading model is adopted, energy consumption is proportional with $d^{4}$.

The energy consumption of receiving $k$ bits data information:

$$
E_{R x}=E_{\text {elec }}(k)
$$

The energy consumption of fusing $k$ bits data information:

$$
E_{F x}=E_{\text {fusion }}(k)
$$

\subsection{The Mathematical Programming Model}

Assume the network has $n$ nodes which needs to be divided into $m$ clusters, and each cluster has a link coming from the nodes in traversal cluster, the square of distance is $C_{i j}$ between nodes $i$ and $j$, the solving goal is to obtain the minimum sum of squares of distance.

Variables are defined as follows:

$$
x_{i j k}= \begin{cases}1 & \text { the No.k link from node } \mathrm{i} \text { to visit node } \mathrm{j} \\ 0 & \text { others }\end{cases}
$$




$$
y_{i k}= \begin{cases}1 & \text { node } \mathrm{i} \text { is on the No.k link } \\ 0 & \text { others }\end{cases}
$$

Mathematical planning model is:

$$
\min (Z)=\min \left(\sum_{k=1}^{m} \sum_{i=1}^{n} \sum_{j=1}^{n} c_{i j} x_{i j k}\right)
$$

S.T:

$$
\begin{aligned}
& \sum_{k=1}^{m} y_{i k}=1 \quad i=1,2, \cdots, n \\
& \sum_{i=1}^{n} x_{i j k}=\left\{\begin{array}{lr}
0 & j \in S ; k=1, \cdots, m \\
y_{j k} & \text { others }
\end{array}\right. \\
& \sum_{j=1}^{n} x_{i j k}=\left\{\begin{array}{lr}
0 & i \in T ; k=1, \cdots, m \\
y_{i k} & \text { others }
\end{array}\right.
\end{aligned}
$$

In the expression, ${ }^{S}$ represents the link starting point set, $r$ represents link endpoint set. In this model, $z$ in expression (4) represents the objective function; constraint (5) guarantees each node access only by one link; Constraints (6) and (7) are used to eliminate subcycle and form the head and end nodes of link.

\section{Solving based on Hybrid Quantum Evolutionary Algorithm}

In order to obtain the optimal solution described by mathematical programming model, this paper improves quantum evolutionary algorithm [8], combines with some concepts of particle swarm optimization algorithm [9] to get a kind of hybrid quantum evolutionary algorithm. This hybrid algorithm can enrich the optimal behavior and process, and increase the ability of searching speed and global search. The improvement of algorithm is mainly from quantum coding and quantum revolving door.

\subsection{Quantum Coding}

Quantum population is defined as $Q(t)=\left\{p_{1}^{t}, p_{2}^{t}, \cdots, p_{v}^{t}\right\}$, hereinto, $t$ is evolutionary algebra, $v_{\text {is }}$ the population scale, $P_{i}^{t}$ represents No. ${ }^{i}$ quantum individual while in No. $t$ generation, at the same time particle swarm algorithm is one of the most optimal individual concept, namely $p_{1}^{\text {best }}, p_{2}^{\text {best }}, \cdots, p_{v}^{\text {best }}$ and $p^{\text {gbest }}$. $p_{i}^{\text {best }}$ and $p^{\text {gbest }}$ represent No. $i$ quantum history optimal individual and population global optimal quantum individual. For simplicity, this paper limits quantum bit probability $\alpha$ and $\beta$ as real, the geometric express of plane quantum bit $\Psi$ is shown as formula (8), and $\Phi$ is the quantum phase angle.

$$
|\psi>=\cos (\varphi)| 0>+\sin (\varphi) \mid 1>
$$


In this paper each quantum individual $P$, including a quantum bit matrix $\mathrm{O}$, a $0-1$ observation matrix $M_{t}$ and a sequence array $S_{t}$. Hereinto, O is a three dimensional matrix by quantum bit. Size is $L \times L \times 2(L=m+n-1)$, it can be divided into two $L \times L$ matrixes, and each of them can be expressed as ${ }^{\alpha}$ and ${ }^{\beta}$ component; $M t$ is the $0-1$ two-dimensional matrix $L \times L$ observed by Martrix $\mathrm{O}$; $S t$ is the sequence array decoded from observation matrix $M t$, length $\mathrm{L}$ is used to express routing link arrangement. The observation and decoding way $\mathrm{O} \rightarrow M t \rightarrow S t$ is as follows.

Considering the normalization condition of $\alpha$ and ${ }^{\beta}$, in the observation process it is simplified as the only operation to ${ }^{\beta}$ component, to adopt the method of "first longitudinal late transverse" to get the observation matrix $M t$ : (1) to generate a temporary component ${ }^{\prime}$ of ${ }^{\beta}$ and the longitudinal stochastic sequence with length of $L ;(2)$ according to the sequence of $S_{q}^{1}$ to do operation on No. ${ }^{i}$ line of ${ }^{\beta^{\prime}}$,then generates a random number $R a$ of ${ }^{[0,1]}$ and lateral random sequence $S_{q}^{2}$ with length of $L$; (3) according to the sequence of $S_{q}^{2}$ in $\beta^{\prime}$ choose corresponding column $j$, from this to observe $\beta_{i j}^{\prime}$, if $\left|\beta_{i j}^{\prime}\right|^{2}$ is greater than random number $R a$, then the corresponding position in the matrix $M t$ is set to 1, at the same time the data of No. ${ }^{i}$ line and No. ${ }^{j}$ column on ${ }^{\beta}$ ' are done the process of returning to zero, and to implement step (4), otherwise choose the next number of $S_{q}^{2}$, repeat step (3); (4) if $S_{q}^{1}$ reaches to end then complete, otherwise jump to step (2), select the next number of $S_{q}^{1}$.The detailed example is illustrated in Figure 1.

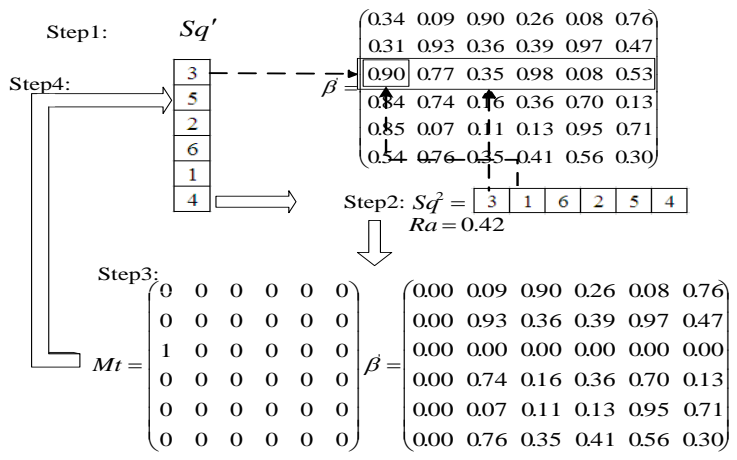

Figure 1. The Example of Observation Steps

\subsection{Update of the Quantum Gate}

In quantum revolution algorithm, the state switch is realized through quantum transforming door. According different problems, there are several quantum transforming door to choose: Exclusive or gate, Hadamard transforming door [10], and revolving door [11]. This paper references the literature [12] and [13], adopts quantum revolving door to realize the state switch. In quantum revolving door, quantum rotation direction and angle are chosen according to the look-up table [13]. Although in the look-up table under different conditions 
the rotation angle is different, but the angle in each case is fixed, it is easy to make quantum population appear "premature" phenomenon or slow convergence speed. This paper improves the adjustment strategy of quantum revolving door, and its core thought is: quantum in evolution will rotate to global optimal quantum $P_{g}^{\text {best }}$ with probability $\mu$, if it does not satisfy the conditions then it will rotate to the optimal $P_{i}^{\text {best }}$ in the history of the quantum. The computation formula of probability is as follows:

$$
\mu=e^{t / T-1}
$$

Hereinto $t$ is the times of current iteration, and $\mathrm{T}$ is the sum of iterations. This makes quantum can keep diversity and rough granularity searching global space in evolutionary initial stage, and in the middle and late stages it rotates gradually toward to the global optimal quantum to guarantee the convergence.

\subsection{Cataclysm}

When the operation gets into the local optimum, the current optimal individual is kept to produce other individuals, steps into the next phase of evolutionary, and jumps out of local optimal solution. In the algorithm the evolution threshold is set, if the continuous not-updated algebra of global optimal value of algorithm succeeds evolution threshold value, then the current optimal individual is kept to initialize other individuals again.

\subsection{Fitness Calculation}

After the update of quantum location every time, quantum individual is used to do observation and decoding to get link coding, and according to formula (4) to calculate the objective function value $Z$, the fitness is:

$$
\text { Fitness }=1 / Z
$$

\subsection{Solving Steps}

The execution procedure of hybrid quantum evolutionary algorithm is as follows. Step 1: initialize quantum population; Step 2: compute each quantum individual fitness value, update

$P_{i}^{\text {best }}$ and $P_{g}^{\text {best }}$; Step 3: execute quantum rotation, update $P_{i}^{t}$ as $P_{i}^{t+1}$; Step 4: determine whether to take catastrophe; Step 5: if conform to the end condition, then exit algorithm and output results; Otherwise return to step 2.

\section{Cluster Head Election Strategy Optimization}

In the cluster head selection stage, many researchers consider to construct the minimum spanning trees [14] which cover all cluster head with the sink as root, or the nearest cluster head link [15-16]. These strategies all adopt multiple-jump way to communicate, and in the hierarchical routing algorithm of clusters it is shorter than single-jump communication length, which has better energy saving effect. But these strategies only consider distance factors, easily cause the distribution disequilibrium situation of cluster head.

This paper improves the election method of cluster head, comprehensively considers of the node own residual energy and the distance to target node, rather than just according to the length of distance to choose cluster head. This breaks invariable pattern about the election of 
cluster head according to the father cluster head, makes cluster head tend to change, and balances the energy consumption of cluster head.

The improved cluster head selection process is, at first, in the network selecting the node as a father cluster head with the largest ratio that residual energy to sink distance (formula (11)), and adding into the cluster head link. Then taking the end nodes in cluster head chain as destination node periodically, searching for the nodes with the maximum right values as the cluster head from clusters which are still not joining in cluster head chain, adding them into the cluster head chain, and eventually form a cluster head link.

Weights calculation formula is:

$$
W_{\text {eight }}=\text { Energy }_{\text {oneself }} / \text { Dis } \tan c e^{2}
$$

In the expression, Energy ${ }_{\text {oneself }}$ is the surplus energy of node, Distance is the distance to target node. Figure 2 is the comparison of network survival period of method A before and after optimization. Method A after optimization its first node's death delays nearly 200 rounds than former, and in general it is near the effect of method B.

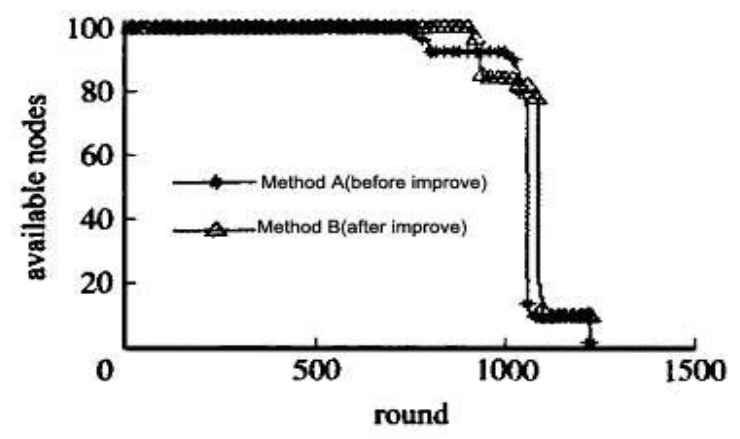

Figure 2. The Comparison of Survival Period Before and After Optimization

\section{The Results Analysis}

In order to evaluate the performance of routing protocol, this paper adopts Matlab to do simulation. In the $100 \mathrm{~m} \times 100 \mathrm{~m}$ square area, randomly 100 nodes are deployed, the number of cluster head is $5 \%$ of the node number, the sink is located at $(50,150)$. Node initial energy is $0.3 J$, hereinto $E_{\text {elec }}$ is set as $50 \mathrm{~nJ} /$ bit,${ }_{f s}$ takes $10 \mathrm{pJ} /\left(\right.$ bit $\left.\times m^{2}\right),{ }_{m p}$ is 0.0013 $\mathrm{pJ} /\left(\mathrm{bit} \times \mathrm{m}^{4}\right), d_{0}$ is $87, E_{\text {fusion }}$ of the data fusion energy is set $5 \mathrm{~nJ} / \mathrm{bit}$, the size of data packet fusion maintains 2000 bit before and after data fusion.

These nodes uses hybrid quantum evolutionary algorithm to calculating optimal routing link, in the simulation the number of quantum populations is set as 20 , the iterative time is 1000 generations, catastrophe threshold is 50 .

With the aid of the proposed hybrid quantum evolutionary algorithm, an optimal link structure is obtained (Figure 3). The links among nodes are very compact, this is also the advantage of apply the intelligent optimization algorithm in this problem. In Figure 3 the sum of square of distance is 7456, in the same situation, the value of PEGASIS is 20573, it is higher than this algorithm. Therefore this kind of link optimization can greatly shorten communication distance among nodes; it is helpful to reduce the energy consumption and lowdown the delay. 


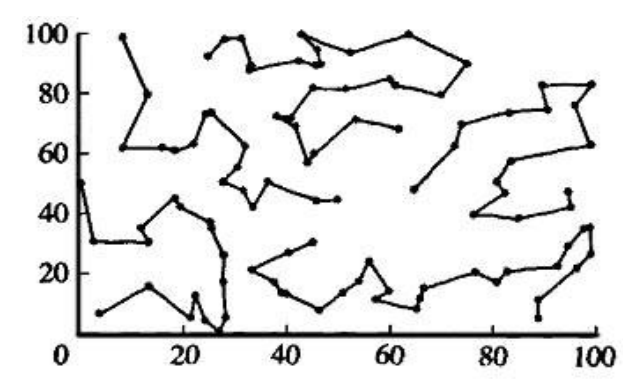

Figure 3. The Network Construct Obtained by this Algorithm

Figure 4 is the life cycle line chart about the networks of ACH, PEGASIS and HERA algorithm, hereinto HERA-1 represents cluster head election using HERA algorithm with minimum spanning tree, HERA-2 represents HERA algorithm after cluster head optimization. From the chart, HERA-1 and HERA-2 compare with LEACH and PEGASIS, they significantly extend the network life, and this is because through hybrid quantum evolutionary algorithm they all get the shortest routing path. In the comparison with same route and different cluster head election, HERA-2 head node death is later about 200 rounds than HERA-1, which indicates optimizing the selection of cluster head with comprehensive consideration of distance and power factor, can better save energy and ensure node energy balance.

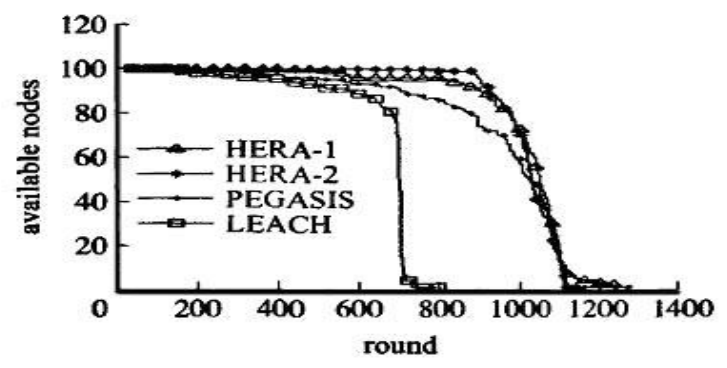

\section{Figure 4. The Comparison of Network Life Cycle}

Figure 5 represents the energy variance of every algorithm and nodes for each round; it reflects the network energy consumption balance. From the diagram PEGASIS and LEACH remains at high position, at the same time the fluctuation is obvious; this indicates their energy consumption is not balanced. The link structure and the cluster head election of HERA-1 and HERA-2 are reasonable, so before a large number of nodes die the energy consumption variance can still maintain at a low position, only in the later period the node's rapid death brings influence and appears higher value. At the same time, comparing with HERA-1 and HERA-2 the cluster head selection is more reasonable, the network energy consumption is more balance. 


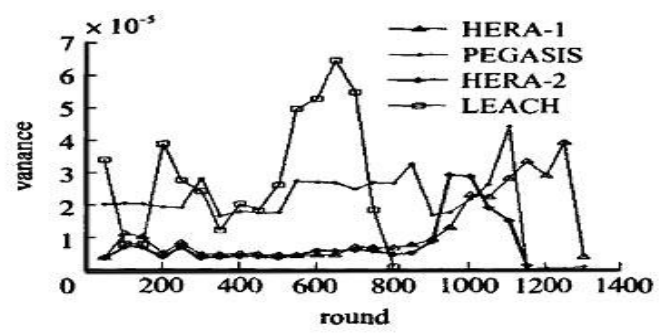

Figure 5. Comparison of Energy Variance

Figure 6 expresses the relationship between the node average network life (half nodes die) and the sink position. In the diagram $\mathrm{x}$ axis coordinates of sink keeps 50. The $\mathrm{Y}$ axis coordinates increases from 150 to 400. From which HERA-1 and HERA-2 with the increase of monitoring area and sink distance the network life reduces the slowest, the reduced amplitude are $47 \%$ and $44 \%$ respectively, and LEACH and PEGASIS are $65 \%$ and $62 \%$ respectively. The average life of HERA-2 nodes is also higher than other algorithms; this means no matter selecting from whether routing link construction or cluster head, the method of HERA-2 is more reasonable.

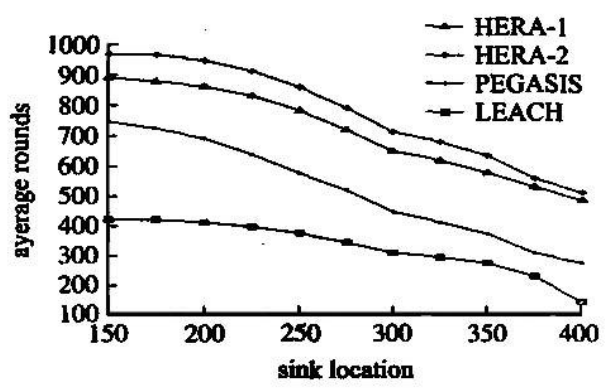

Figure 6. The Influence of Network Life Time with Different Sink Position

\section{Summary}

This paper proposes a kind of high efficiency and energy-saving of wireless sensor network clustering routing algorithm HERA based on hybrid quantum evolutionary algorithm. In this algorithm, the network is divided into several clusters; the nodes in the clusters connect into a multiple jump communication link, and hybrid quantum evolutionary algorithm is used to get the optimal clustering group chain method, which makes the total distance of link shortest. In the communication between cluster head and sink, in order to further reduce energy consumption and maintain node energy equilibrium, using the method of comparing node residual energy and target distance to select cluster head, via multiplejumps through other cluster head to transfer the collected data to sink. Simulation experiments show that HERA can prolong the working life of nodes and network, and maintain node energy balance, and at the same time, in the condition of sink changing which also can maintain good performance. Because in the condition with certain distance, the position of sink will produce great influence to the work life of WSN, in the next work, according to the position of sink to dynamically build routing network will be considered, this kind of influence should be reduced to minimum. 


\section{References}

[1] J. N. Ai-Karaki and A. E. Kamal, "Routing Techniques in Wireless Sensor Networks: A Survey", IEEE Wireless Communications, vol. 11, no. 6, (2004), pp. 6-18.

[2] W. B. Heinzelman, A. P. Chandrakasan and H. Balakrishnan, "An Application Specific Protocol Architecture for Wireless Microsensor Networks", IEEE Trans on Wireless Communications, vol. 1, no. 4, (2002), pp. 660-670.

[3] S. Lindsey and C. S. Raghavendra, "PEGASIS: Power Efficient Gathering in Sensor Information Systems", Aerospace Conference Proceedings, vol. 3, (2002), pp. 1125-1130.

[4] N. Ding and X. P. Liu, "Data Gathering Communication in Wireless Sensor Networks Using Ant Colony Optimization", Proceedings of the IEEE Conference on Robotics and Biomimetics, Shenyang, China, (2004), pp. 822-827.

[5] S. H. Lee, J. J. Yoo and T. C. Chung, "Distance Based Energy Efficient Clustering for Wireless Sensor Networks", Proceedings of the 29th Annual IEEE International Conference on Local Computer Networks (LCN 04), (2004).

[6] M. Liu, H. Gong and Y. Mao, "The high efficiency and energy-saving sensor network data collection and polymerization agreement", Journal of Software, vol. 16, no. 12, (2005), pp. 2106-2116.

[7] F. Huang and X. Jin, "The WSN routing protocol based on GASA energy equilibrium", Journal of Sensing Technology, vol. 22, no. 4, (2009), pp. 586-592.

[8] K. H. Han and J. H. Kim, "Quantum Inspired Evolutionary Algorithm for a Class of Combinational Optimization”, IEEE Transactions on Evolutionary Computing, vol. 6, no. 6, (2002), pp. 580-593.

[9] H. Demir, A. Ozturk, L. Kuru and E. Kuru, "Weight optimization of a dry type core form transformer by using particle swarm optimization algorithm", Energy Education Science and Technology Part A., vol. 29, no. 2, (2012), pp. 1063-1072.

[10] T. Hey, "Quantum Computing: An Introduction", Computing \& Control Engineering Journal, vol. 10, no. 3, (1999), pp. 105-112.

[11] L. Wang, H. Wu and F. Tang, "A Hybrid Quantum Inspired Genetic Algorithm for Flow Shop Scheduling”, Advances in Intelligent Computing International Conference on Intelligent Computing, (2005), pp. 636-644.

[12] Y. Zhao and D. Peng, "The quantum evolutionary algorithm with capability constraint about vehicle routing problem”, Journal of System Engineering Theory and Practice, vol. 29, no. 2, (2009), pp. 159-166.

[13] X. Huanlai and L. Xin, “A Multi Granularity Evolution Based Quantum Genetic Algorithm for QoS Nulticast Routing Problem in WDM Networks", Computer Communications, vol. 32, (2009), pp. 386-393.

[14] Y. Shen, W. Qi and H. Dai, "A kind of energy-effective sensor monitoring network fascicles agreement", Journal of Software, vol. 19, no. 9, (2008), pp. 2432-2441.

[15] Q. Tang and T. Tao, "Research of LEACH routing algorithm based on the minimum spanning tree", Computer Technology and Development, vol. 19, no. 4, (2009), pp. 109-111.

[16] Y. Xie, X. Xie and Y. Zhang, "The hierarchical wireless sensor network protocol based on Dijkstra algorithm", Journal of Sensing Technology, vol. 22, no. 2, (2009), pp. 249-253.

\section{Authors}

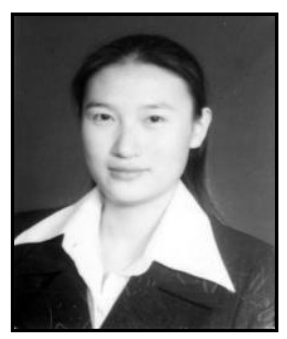

Lingxia Liu was born in 1976.5, she incepted her master degree in Shanxi University in 2003, and her major is information technology.

Now she is working in Computer Education Department of Anyang Normal University as an associate professor. She is also a member of China Computer Federation. 


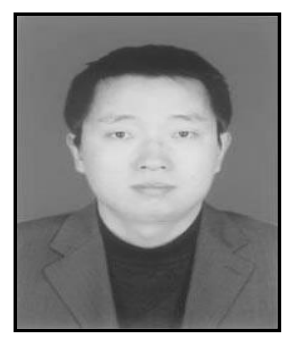

Qiang Song was born in 1971.2, he incepted his double master degrees in Ateneo de Manila University in the Philippines in 2005, which are MSIT and MBA. Now he is a PhD. Candidate in Wuhan University of Technology in major of information engineering.

$\mathrm{He}$ is working in Anyang Institute of Technology as a Professor, and he is also a senior member of China Computer Federation. 